\title{
ENHANCEMENT OF BAYESIAN MODEL WITH RELEVANCE FEEDBACK FOR IMPROVING DIAGNOSTIC MODEL
}

\author{
Fatihah Mohd ${ }^{1}$, Masita Abdul Jalil ${ }^{1}$, Noor Maizura Mohamad Noor', Zainab Abu Bakar', Zailani Abdullah ${ }^{3}$ \\ ${ }^{1}$ School of Informatics and Applied Mathematics, Universiti Malaysia Terengganu \\ 21030 Kuala Terengganu, Terengganu, Malaysia \\ ${ }^{2}$ Faculty of Computer and Information Technology, Al-Madinah International University \\ Shah Alam, Selangor, Malaysia \\ ${ }^{3}$ Faculty of Entrepreneurship and Business (FEB) / Centre of Computing \& Informatics (CCI), UMK, \\ City Campus, Kota Bharu, Kelantan \\ Email: mpfatihah@umt.edu.my.com ${ }^{1}$
}

DOI: https://doi.org/10.22452/mjcs.sp2018no1.1

\begin{abstract}
An enhanced method to classify multi-class clinical disease is proposed in this study. The enhanced method is based on the Bayesian Model, which incorporates Bayes' rule and probability theory. It covers three main components: prior, conditional, and posterior probability. The recommended enhancement method is the Bayesian Relevance Feedback (BRF) Model. BRF can solve the non-existent value of posterior probabilities (zero values of probability), focusing on increasing the classification accuracy in the diagnosis of disease. The BRF has the capability to produce significant classes or target (cancer stage) by exploiting relevance feedback. Consequently, models based on eight different classifiers-K-Nearest Neighbors, Bayesian Model, Rule OneR, Meta MultiClass Classifier, Multilayer Perceptron, Random Tree, SMO-Poly Kernel, and Naive Bayes-were applied in the evaluation process. The results of the experimental works using an oral cancer dataset show that BRF outperformed the eight other classifier models, achieving 95.83\% classification accuracy.
\end{abstract}

\section{Keywords: $\quad$ Bayesian model, Bayesian relevance feedback, classification, clinical diagnosis, oral cancer.}

\subsection{INTRODUCTION}

The accurate identification and diagnosis of cancer disease is crucial in medical practice, especially when predicting the stage of cancer [1], [2]. When predicting cancer, a friendly and accurate diagnosis system is essential to support patient survival [3]. Currently, the diagnosis and treatment of cancer patients rely on the cancer staging system. According to the American Cancer Society (ACS), the stage of cancer is the primary indicator of whether or not the patient would have to suffer and live with cancer throughout his or her entire life [4]. Collaborated treatment delivered by various expert groups is essential to maximize the patient's prognosis and survival, as almost all types of cancer show no symptoms at the beginning. Most first-stage cancer patients cannot be traced further. Therefore, an accurate disease diagnostic model needs to be developed to predict survival and identify appropriate treatment strategies for patients before the start of any treatment [3], [5].

Past studies have shown that Artificial Intelligence (AI) can be used with machine learning (ML) techniques to improve diagnostic accuracy [6], [7], [8], [9], [10]. These techniques, which can take many forms, such as Bayesian, Neural Network, and Fuzzy methods, have been implemented in applications to solve uncertainty problems. Some of the AI and ML methods are more frequently used in diagnostic models [11]. The Bayesian Model (BM) has gradually been applied in various domains for adapting disease classifications [12]. BM is capable of integrating relevant external knowledge to assist in making a more meaningful conclusion [13]. Many studies have indicated that BM has the ability to increase the level of system accuracy [13], [14], [15], [16], [17]. Based on numerous AI applications in various areas, BM has been acknowledged as a dominant approach in the learning process and has been implemented in model-based relevance feedback [18], [19]. The other benefit of the learning processes based on relevance feedback, or also known as the iterative model, is its capability to improve the performance of classification domain accuracy [20], [21], [22]. 
The accurate computation of differential probabilities of multiple class labels and new cases is becoming more challenging. This issue requires a method that can handle similarity parameters among different cases as well as expert knowledge. This has motivated this research to explore options for a new diagnostic model that would focus on accuracy improvement. Therefore, this study will implement an AI method in the development of a diagnostic model for disease classification. This enhanced model will be applied to generate a significant diagnostic result.

In this study, an enhanced inference engine (IE) model is proposed to generate more accurate diagnosis of cancer stages based on BM. The collected dataset of oral cancer (OCDS) from prior research was used utilizing a BMbased iteration for stage classification [23], [24]. By enhancing the BM, the study developed a Bayesian Relevance Feedback Model (BRF) to produce differential probabilities of oral cancer $(\mathrm{OC})$ diagnosis. This model can also deal with new cases and then combine them with expert knowledge to generate a new diagnosis probability.

This paper is organized into six sections: in Section 2, related works on the issue is presented. Section 3 describes the diagnosis of oral cancer, the case study, the disease and its symptoms and signs, as well as the differential diagnoses. The basic principles of the inference engine and the Bayesian model are discussed in Section 4. Section 5 presents the proposed implementation for diagnosis of the case study datasets and discusses all the corresponding results. Lastly, the conclusion of the study is drawn in Section 6, based on the findings.

\subsection{RELATED WORKS}

Many medical researchers have applied the Bayesian model (BM) in multiple domains such as medical diagnostics [25], classification [26], visualization [27], and treatment planning [28]. In the diagnosis of a disease, the Bayesian inference engine model has been applied to calculate probability analysis. This model applies the Bayes' theorem, which was proposed by Thomas Bayes [29].

In previous studies, BM was also developed to predict the malignancy of thyroid nodules, and sentinel lymph nodes [30]. The prediction process was not easy for oncologists, even after mapping and performing the biopsy [31]. Another study applied Bayesian in a decision support system for supporting vitamin D shortage management [32]. BM was also found capable of improving the accuracy of medical diagnostic systems and in supporting complex clinical diagnoses [33].

From the literature, inference engine methods that have been used in a clinical decision support system (CDSS) include rule-based, Bayesian, probabilistic programming, adaptive neurofuzzy, heuristics, neural network, and Genetic Algorithm. The inference mechanism method can be used on its own or combined with several methods to form a hybrid system [3], [34]. Kose et al. [35] presented a medical diagnosis system with a hybrid inference model that combined the Bayesian Network and rule-based method. The performance evaluation of the system was presented via three tests: Rule-based method, Bayesian Network-based method, and a linear combination of both methods. The findings of the study showed that the linear combination produced more accurate results than the other two methods. The success rate increased to $80 \%$, much higher than the values obtained by applying the Rule-based (77.82\%) and Bayesian (52.36\%) methods only.

In another field, a Bayesian-inference-based recommendation system was presented for social media. The ranking similarity of two users was quantified using a set of conditional probabilities taken from the users' past mutual ranking. The study suggested the use of prior distribution to overcome early humidity and rare rating. The evaluation of the proposed algorithm was presented via two different sets of online rating with real user datasets. The proposed model was found to be better than previous belief-based recommendations and was as good as the cooperative filtering recommendation. It also tolerated the flexibility between quality and quantity recommendation [36].

\subsection{APPLICATION CONTEXT: DIAGNOSIS OF ORAL CANCER}

The clinical diagnosis process starts with the gathering of patient information from history records. The clinical diagnosis involves a few steps. It begins with patient history derived from a main record of disease signs, previous health records, and the background of social life and relatives [37], [38]. This is followed by physical examination, where the doctor will be able to identify abnormalities by seeing, feeling, and hearing all parts of the patient's body. In the next process, the pathologist will conduct further investigations with blood tests, a biopsy, and x-ray sample analysis. In this step, sometimes, the doctor could make early decisions by formulating a list of possible diagnoses. 
Therefore, a list of differential diagnoses, arranged from most likely to least likely, could be generated, enabling the doctor to provide a plan for further diagnostic testing and treatment [37].

Identifying the stage of oral cancer is important, so that the right treatment can be started and a prognosis determined. Tumor is staged using the Tumor, Node, Metastasis (TNM) staging system, where T denotes the size of the primary tumor, $\mathrm{N}$ indicates the status of the regional lymph nodes, and $\mathrm{M}$ represents the presence or absence of distant metastases (see Table 1). This tumor is defined in the TNM staging system (see Table 2) [39], [40], [41].

Table 1: TNM description

\begin{tabular}{|l|l|}
\hline TNM & Description \\
\hline T1 & Tumor is two cm or less in greatest dimension \\
T3 & Tumor is more than two cm but is not more than four cm in greatest dimension \\
T4 & Tumor is more than four cm in greatest dimension \\
& Tumor invades through cortical bone, inferior alveolar nerve, floor of mouth, or skin (chin or \\
& Tumor invades through cortical bone, into the deep or extrinsic muscle of the tongue \\
& (genioglossus, hyoglossus, palatoglossus, and tyloglossus), maxillary sinus, or skin of the \\
& face \\
& Tumor invades masticator space, pterygoid plates, or skull base or encases internal carotid \\
N0 & No regional lymph node metastasis \\
N1 & Metastasis in a single ipsilateral lymph node, three cm or less in greatest dimension \\
N2 & Metastasis in a single ipsilateral lymph node is more than three cm but is not more than six \\
& cm in greatest dimension \\
& Metastasis in multiple ipsilateral lymph nodes, none more than six cm in greatest dimension \\
& Metastasis in bilateral or contralateral lymph nodes, is not more than six cm in greatest \\
N3 & dimension \\
Metastasis in a lymph node is more than six cm in greatest dimension \\
M1
\end{tabular}

Table 2: TNM staging system

\begin{tabular}{|l|l|l|l|}
\hline Stage & T & N & M \\
\hline Stage I & T1 & N0 & M0 \\
Stage II & T2 & N0 & M0 \\
Stage III & T1, T2, & N1 & M0 \\
& T3 & N0, N1 & M0 \\
Stage IV & T1, T2, T3 & N2 & M0 \\
& T4 & N0, N1, N2 & M0 \\
& Any T & N3 & M0 \\
& T4 & Any N & M0 \\
& Any T & Any N & M1 \\
\hline
\end{tabular}


In the following sections, a brief description of the Bayesian model, which is used as the base model in this study, is given. Then, the proposed model, BRF, its main components, and its process are discussed.

\subsection{BAYESIAN INFERENCE/THEOREM}

The Bayesian Model (BM) is a simple probabilistic model built from Bayes' theorem (or Bayes' rule), which has three main components: prior, conditional, and posterior probability [42]. The original concept of Bayes' rule is that the outcome of an event $(A)$ can be predicted based on some evidences $(B)$ that can be observed. Bayes' rule presents a prior probability of $A$ or $P(A)$, which means the probability of an event before the evidence is observed, and a posterior probability of $A$ or $P(A \mid B)$, which means the probability of an event after the evidence is observed. These tree main components of BM are applied in the proposed model of this study. This could be represented by Eq. (1):

$$
P(A \mid B)=\frac{P(B \mid A) \times P(A)}{P(B)}
$$

Where: $P(A \mid B)$ is the class (target) posterior probability given features, $P(A)$ is the class prior probability, $P(B \mid A)$ is the likelihood, which is the features probability given class, and $P(B)$ is the features prior probability.

In the Bayesian inference engine (IE), the finding of posterior probabilities is assigned as differential probabilities and as a result of the classification of disease. The ranking of the disease probabilities is obtained from the values of posterior probabilities from the highest to lowest order, known as differential probabilities. The differential probability with the highest value will be identified as the disease class (target). The Bayesian model, as outlined in Eq. (1), is the base model for this study. The model can also be applied in multiple events of $A_{j}, j=1,2 \ldots m$ and multiple events of $B_{i}, i=1,2 \ldots n$. This depends on the conditional probability of all possible combinations of $B$ for all $A$ as represented by Eq. (2):

$$
P\left(A_{j} \mid B_{1}, B_{2}, \ldots B_{n}\right)=\frac{\prod_{i=1}^{n} P\left(B_{i} \mid A_{j}\right) \times P\left(A_{j}\right)}{\sum_{k=1}^{m} \prod_{i=1}^{n} P\left(B_{i} \mid A_{k}\right) \times P\left(A_{k}\right)}, j=1 \ldots n
$$

This research applies the BM to generate an event given a set of evidences. By utilizing a BM and the probability theory, it produces a prior, conditional, and posterior probability. However, there is a limitation with the BM. The posterior probability will be zero if a feature's value of a new case or given sample does not fit its training set. The experiments conducted with the Bayesian model show some findings with zero conditional probabilities. This scenario will produce a zero posterior probability. In medical diagnosis, the zero posterior probability will lead to a non-diagnosis finding [43].

\subsection{BAYESIAN RELEVANCE FEEDBACK MODEL}

Intelligent Computing Methods (ICMs) is now an increasingly popular method for generating an inference engine (IE) to assist in the development of clinical decision support systems. The Bayesian model, as one of the ICMs, is seen as an appropriate method for generating a diagnostic model. However, issues relating to the lack of diagnosis suggest that an alternative solution should be used, as this could improve the base model selection. With proper improvement, the base model could be processed further, namely by enhancing the BM. In order to ensure that the performance of the clinical diagnostic system will yield improved quality output, it is important to extend the IE model with an enhanced Bayesian diagnostic model. This study presents an enhanced IE clinical diagnosis model based on BM, as presented in Eq. (2), which can adaptively enhance oral cancer diagnosis performance. 
The proposed model comprises three components: prior, conditional, and posterior probability. This study concentrates on posterior calculations to classify stages according to the same oral cancer cases and symptoms. The process of learning in a relevance feedback is adapted in the posterior computation. This section presents the proposed enhanced IE with Bayesian Relevance Feedback Model (BRF) in order to improve the performance of the system and to verify the system's accuracy. The relevant feedback process starts with the system classifying a number of new objects using existing parameters. Since the classification of the object may be correct or incorrect, knowledge experts will be involved in monitoring newly classified objects to improve classification performance and produce high quality results. At the end of each iteration, the model acquires the appropriate class as generated by the expert, and the corrected object feature combined with their class will be applied in the new iteration [26].

In the case of OCDS, the set of features of OC diagnosis is elaborated in Eq. (2). In this study, the four types of OC stages are demonstrated as $A_{j}, j=1,2,3,4$, while the OCDS features with fifteen numbers are represented by $B_{n}, n=1$, $2 \ldots 15$. The stage probability, with the multiple features $B$, is similar to the probability of $B$ expecting that the cancer stage presents, multiplied by the summation probability of existence of the features $B$, in which the cancer stage presents. In this study, 15 features, used in the diagnosis of cancer, are split into two groups. General features consisting of 12 clinical features is referred as $C$ : sex, ethnicity, ulcers, neck swelling, pain, numbness, swelling, size, site, lymph nodes, histological type, and the type of squamous cell carcinoma. Otherwise, three histopathological features are called and the supervise features are assigned as $H$ : primary tumor (T), regional lymph nodes (N), and metastasis (M). Then, the sample summation of $H$ and $C$ posterior is computed and represented as $e$. The steps for the whole process of BRF are explained below:

- First, a set of properly classified OCDS is determined as the training set. Fifteen OCDS features are represented as numerical vectors.

- Then, a prior on knowledge parameters (stage) is calculated.

- After that, the Bayesian rule is employed to calculate the conditional probability of every parameter $B$ and every stage $A$.

- This is followed with defining a general feature as $C$ and a supervise feature as $H$.

- Then, the posterior probability for $C$ and $H$ is computed to identify the class of a new case, as presented by Eqs. (3) and (4):

$$
\begin{gathered}
P\left(C_{i} \mid A_{j}\right)=\frac{P\left(A_{j}\right) \prod_{i=1}^{n} P\left(C_{i} \mid A_{j}\right)}{\sum_{k=1}^{m} P\left(A_{k}\right) \prod_{i=1}^{n} P\left(C_{i} \mid A_{k}\right)}, j=1 \ldots n \\
P\left(H_{i} \mid A_{j}\right)=\frac{P\left(A_{j}\right) \prod_{i=1}^{n} P\left(H_{i} \mid A_{j}\right)}{\sum_{k=1}^{m} P\left(A_{k}\right) \prod_{i=1}^{n} P\left(H_{i} \mid A_{k}\right)}, j=1 \ldots n
\end{gathered}
$$

- After that, the summation of $C$ and $H$ posterior is computed using Eq. (5). In this process, if the posterior value is not zero $\left(\sum e_{i} \neq 0\right)$, then the relevant feedback process is not implemented, and the algorithm of the $\mathrm{BRF}$ is completed.

$$
P\left(e_{i} \mid A_{j}\right)=\frac{P\left(C_{j}\right)+P\left(H_{j}\right)}{\sum_{j=1}^{4} P\left(C_{j}\right)+P\left(H_{j}\right)}, j=1 \ldots n
$$


- Otherwise, if the posterior value is zero $\left(\sum e_{i}=0\right)$, then, a knowledge expert of the TNM staging system presented in Table 2 will replace the zero diagnosis with new class information, which will be used in subsequent iterations. Simultaneously, the posterior of the cancer stage in the current iteration is used as an advanced knowledge level for the subsequent iteration. Next, the process continues with the learning process until a new object is successfully classified.

Then, the proposed BRF is executed by the main procedure of the Bayesian, as shown in Algorithm 1. At line 9, if the value of $e$ is zero, then the procedure to call Algorithm 2 will be executed at line 13. Algorithm 2 is developed based on supervise features, as presented in Table 2.

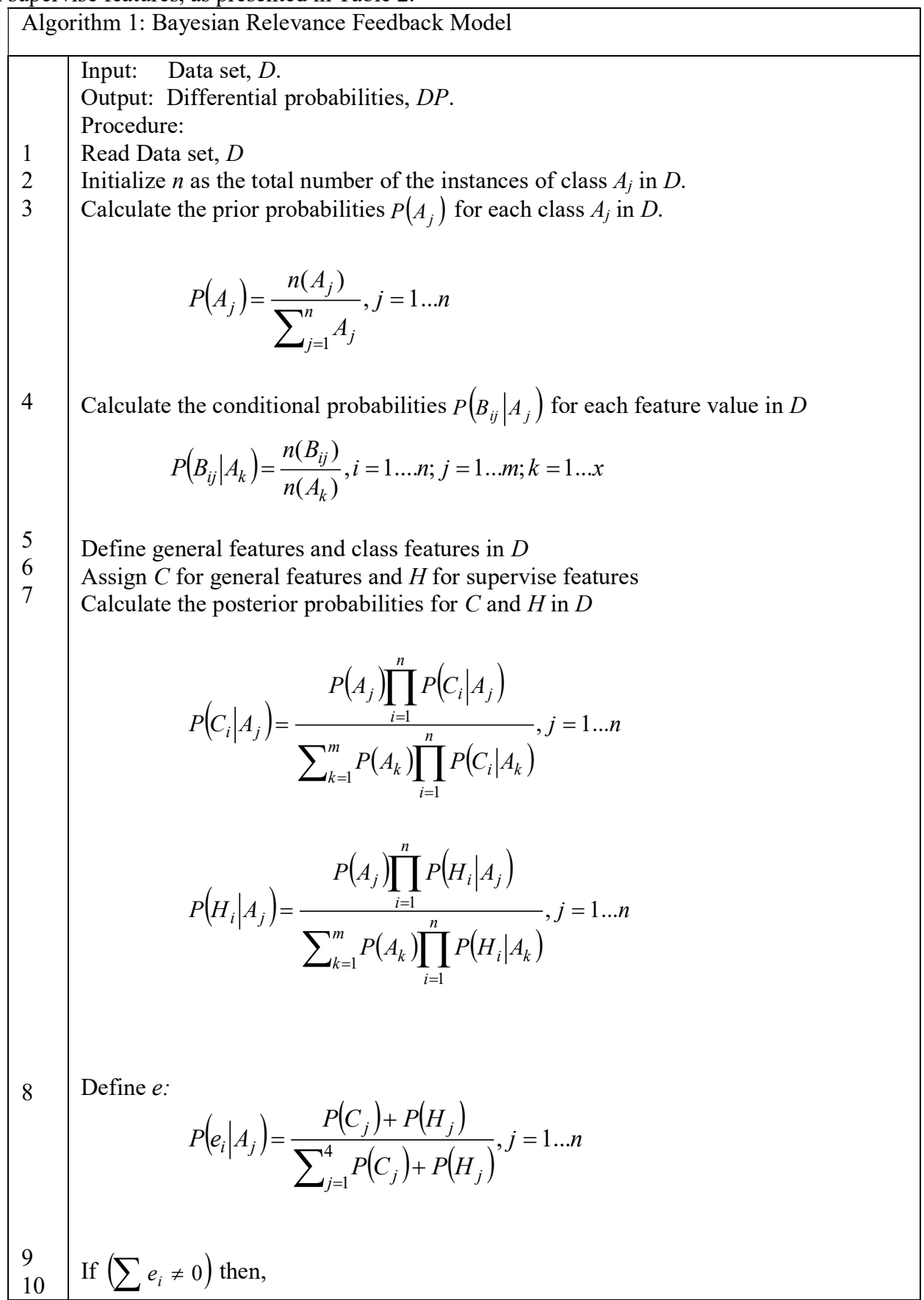




\begin{tabular}{|l|l|}
\hline \multicolumn{2}{|c|}{ Algorithm 1: Bayesian Relevance Feedback Model } \\
\hline 11 & Add new object to D \\
12 & STOP \\
13 & Else Assign class for new object \\
14 & Procedure: Assign new object $(*$ call Algorithm 2$)$ \\
15 & i. Read value $T, N, M$ for new object \\
16 & ii. Assign class value, stage \\
17 & iii. New object correctly classified \\
18 & Add new object to D \\
\hline
\end{tabular}

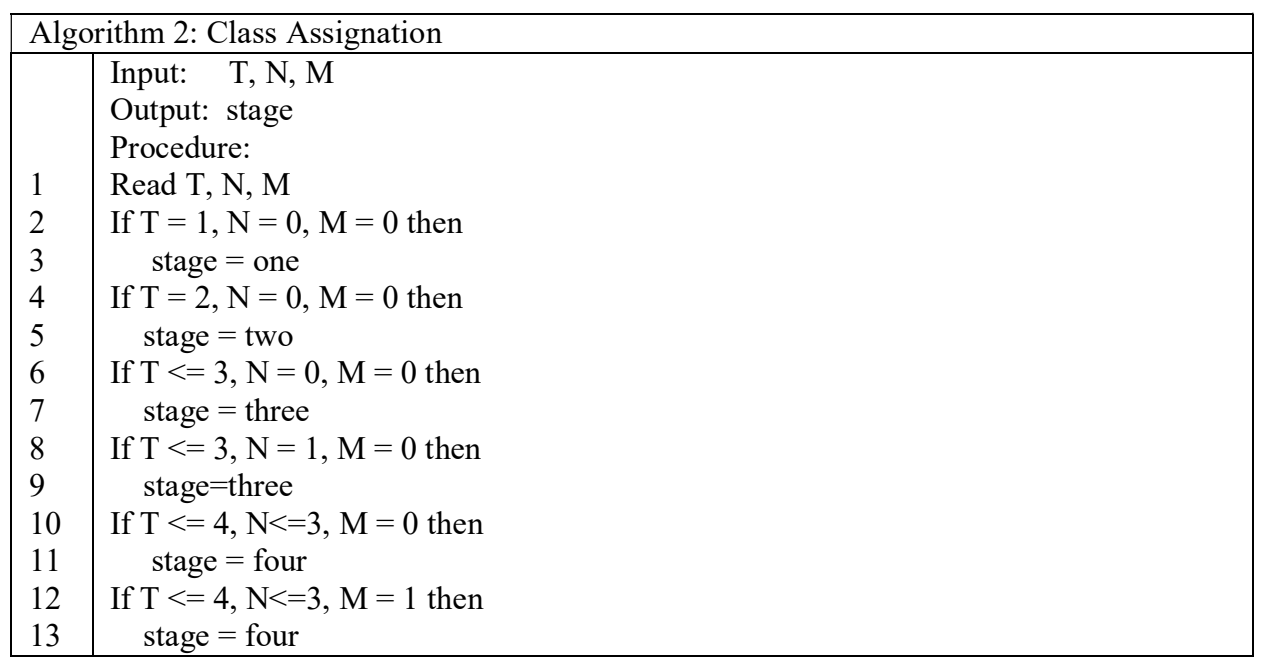

\subsection{EXPERIMENTAL WORK AND EVALUATION}

The Oral cancer dataset (OCDS) used in this case study was developed from a random record review of patients from the Otorhinolaryngology Clinic [23]. The purpose of the experimental works are to diagnose the oral cancer stage. The works involve OCDS with 160 instances and fifteen characteristics. The label of the class (the cancer stage) is divided into classes one, two, three, and four. In demonstrating the BRF, OCDS is split into a training set with 112 instances and a testing set with 48 samples of patients with OC applied in the experimental works.

In this study, performance evaluation was done by comparing the basic Bayesian model (BM) and the proposed model, the BRF. Furthermore, the evaluation of BRF and machine learning (ML) models was also demonstrated. The classification performance was determined using an accuracy unit matrix. The ML experiments were implemented in open source data mining software, Waikato Environment Knowledge Explorer (WEKA) with 10fold cross validation using 48 samples for the test set. The following are the seven (7) different ML models and the scheme of each:

i. Meta MultiClass Classifier (MCC) is an algorithm that takes multi-class datasets with two-class classifiers. MCC is able to remove error and replace it with accurate output codes for improved accuracy.

- weka.classifiers.meta.MultiClassClassifier

ii. K-Nearest Neighbor classifier $(\mathrm{KNN})$ with a value of $\mathrm{K}=1$

- weka.classifiers.lazy.Ibk

iii. Rule OneR (OneR) is a classifier for developing and applying the $1 \mathrm{R}$ algorithm, and it employs the minimumerror feature for prediction and discretizing numeric features.

- weka.classifiers.rules.OneR 
iv. Random Tree (RT) is a classifier for developing a tree that recognizes K based on selected attributes at every node. It performs no pruning. RT also has an option to allow estimation of class probabilities (or target mean in the regression case) based on a hold-out set (back fitting).

- $\quad$ weka.classifiers.trees.RandomTree

v. The Multilayer Perceptron (MLP) classifier applies network back-propagation to classify events. The network of MLP can be developed by hand, generated by an algorithm, or both. The network is observed and changes throughout the training period. All MLP nodes are all sigmoid.

- weka.classifiers.functions.MultilayerPerceptron

vi. Naive-Bayes (NB) is a classification method employing estimator classes. Precision values of the numeric estimator are selected from the trained data analysis. For this reason, the classifier is not an UpdateableClassifier (which, in typical usage, are initialized with zero training instances).

- weka.classifiers.bayes.NaiveBayesUpdateable

vii. Support Vector Machines (SVM) are used widely to substitute absent values; SVMs change nominal features into binary ones and standardize all features automatically.

- $\quad$ weka.classifiers.functions.supportVector.PolyKernel

Accuracy evaluation is a common measure that is used in diagnostic tests [44]. Therefore, the evaluation phase is important for improving the diagnostic model. It helps to discover the most suitable method to represent the domain and ensures the successful operation of the proposed model in the future. The correctness of the classification is measured by the total precision of the model and is computed as the overall correctness divided by the total number of correct classification. Besides that, the error rate, sensitivity, and specificity evaluation of the model using OCDS are also determined in this study [45], [46]. The performance of classifiers is presented as a classification confusion matrix (see Table 3) with the following items: TN for accurate predictions when the given instance is negative, FP for the error predictions when a given instance is positive (FP), FN indicating the error predictions when a given instance is negative, and TP for the accurate predictions when a given instance is positive.

Table 3: Confusion Matrix

\begin{tabular}{|l|l|l|l|}
\hline \multicolumn{2}{|c|}{} & \multicolumn{2}{c|}{ Actual } \\
\cline { 3 - 4 } & Yes $(A)$ & No (B) \\
\hline \multirow{3}{*}{ Predicted } & Yes $(A)$ & TP & FN \\
& No (B) & FP & TN \\
\hline
\end{tabular}

Using the confusion matrix in Table 3, three criteria for the classification of model performance are applied:

i Sensitivity is proportional? Measure true positives and its flattering false negative rates using Eq. (6).

$$
\text { Sensitivit } y(T P R)=\frac{T P}{(T P+F N)}
$$

ii Specificity is a measure of the true negatives that are correctly identified as such and is flattering to the false positive rate, defined by Eq. (7).

$$
\text { Specificit } y(T N R)=\frac{T N}{(T N+F P)}
$$

iii Accuracy is the precision degree of a classification and is identified from measures of specificity and sensitivity. The most efficient predictor would be described as $100 \%$ sensitive and $100 \%$ specific, and given by Eq. (8).

$$
\text { Accuracy }=\frac{T P+T N}{(T P+F P+T N+F N)}
$$


The values of FN, TP, FP, and TN as well as the accuracy, error, sensitivity, and specificity of the eight ML models, were obtained from experimental works using WEKA classification. Meanwhile, BFR was evaluated based on the confusion matrix.

\subsection{RESULT AND DISCUSSION}

In this section, the experimental findings of BRF and the eight ML models are presented. Table 4 shows the model performance for the test data in regard to the accuracy, error rate, sensitivity, and specificity of the nine models. The findings indicate that BRF yielded the highest accuracy of $95.83 \%$ and the lowest error rate (4.17\%) compared to the rest of the models. BRF also gave the highest value of specificity and sensitivity $(93.9 \%$ and $93.8 \%$, respectively). Two models also achieved similar values of accuracy (93.75\%) i.e. NB and SMO, while the Bayesian base model only gave $74.94 \%$ accuracy, which is the lowest performance accuracy.

Table 4: Accuracy, error, specificity, and sensitivity performance of the nine models using OCDS

\begin{tabular}{|l|l|l|l|l|}
\hline Method & Accuracy & Error & Sensitivity & Specificity \\
\hline BM & 74.94 & 25.06 & 91.67 & 62.50 \\
KNN & 83.33 & 16.67 & 84.70 & 83.30 \\
MCC & 87.50 & 12.50 & 87.20 & 87.50 \\
OneR & 87.50 & 12.50 & 88.50 & 87.50 \\
RT & 87.50 & 12.50 & 89.60 & 87.50 \\
MLP & 89.58 & 10.42 & 89.80 & 89.60 \\
NB & 93.75 & 6.25 & 93.90 & 93.80 \\
SMO & 93.75 & 6.25 & 93.90 & 93.80 \\
BRF & 95.83 & 4.17 & 95.80 & 96.40 \\
\hline Summary: \\
Bayesian Model (BM), K-Nearest Neighbor (KNN), Meta MultiClass Classifier \\
(MCC), Rule OneR (OneR), Random Tree (RT), Multilayer Perceptron(MLP), \\
Naive-Bayes (NB), SMO-Poly Kernel: E-1.0 (SMO), Bayesian Relevance \\
Feedback (BRF).
\end{tabular}

The nine comprehensive evaluation models used in the diagnosis of oral cancer are illustrated in Fig. 1 and Fig. 2. The findings presented in Fig. 1 show that accuracy rate increased skewing to the right with a greater number of states. The classification accuracy performed by the eight ML models ranged from $74.94 \%$ to $93.75 \%$. Interestingly enough, BRF outperformed the other models, achieving $95.83 \%$ accuracy, correlating to significantly improved accuracy by $20.89 \%$ from the BM performance of $74.94 \%$. 


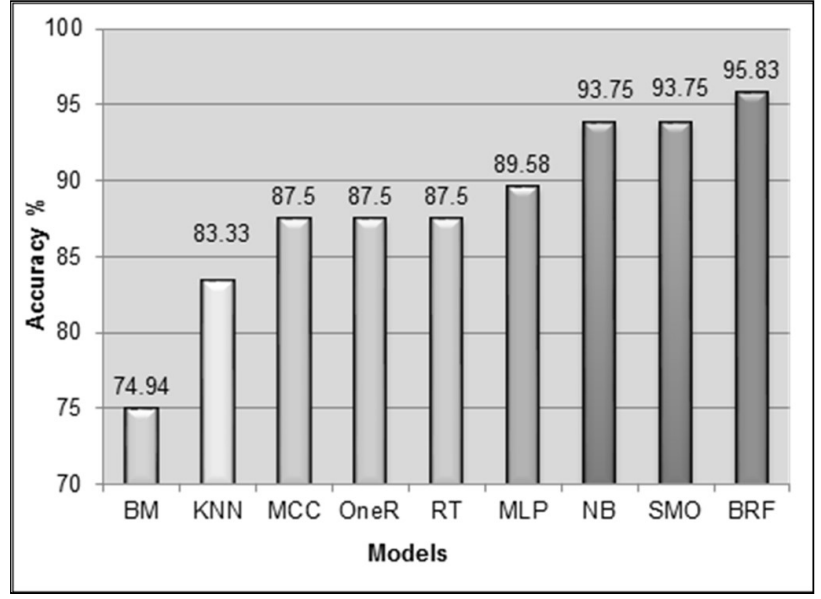

Fig. 1. The performance of BRF in terms of accuracy compared to the eight selected ML models

Fig. 2 presents the error rate performance of the BRF and eight ML models. BRF showed the lowest error rate with only $4.17 \%$. SMO and NB showed the same error rate value (6.25\%), while OneR, MCC, and RT also shared a similar error rate of $12.5 \%$. The finding of experimental works demonstrates that the highest error rate was observed with the use of BM, at $25.06 \%$. From the experimental work, BRF was able to obtain higher accuracy for the OCDS classification. Therefore, BRF was the best diagnostic model compared to the eight ML models.

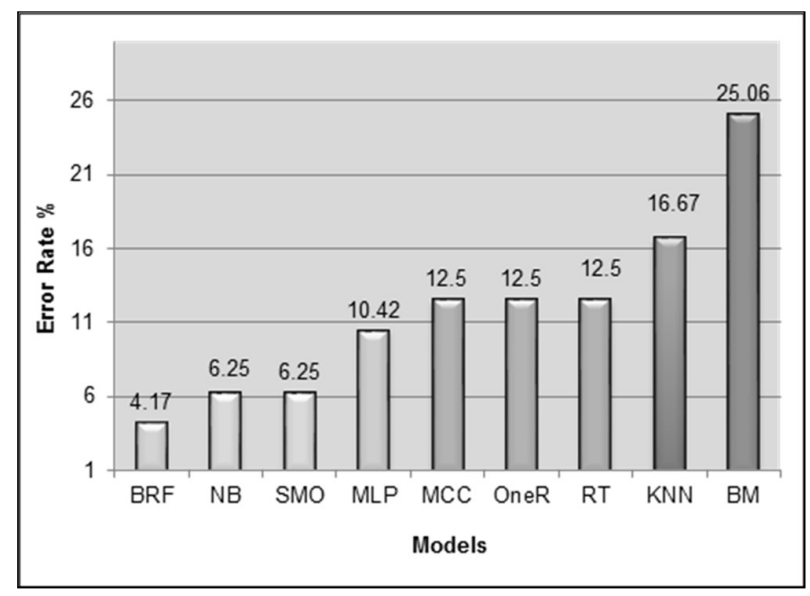

Fig. 2: Performance of BRF error rate compared to eight ML models

From the results illustrated in Fig. 3 and Fig. 4, the sensitivity and specificity of BRF outperformed the other ML models. BRF also successfully increased the rate of sensitivity (95.8\%) and specificity (96.4\%). 


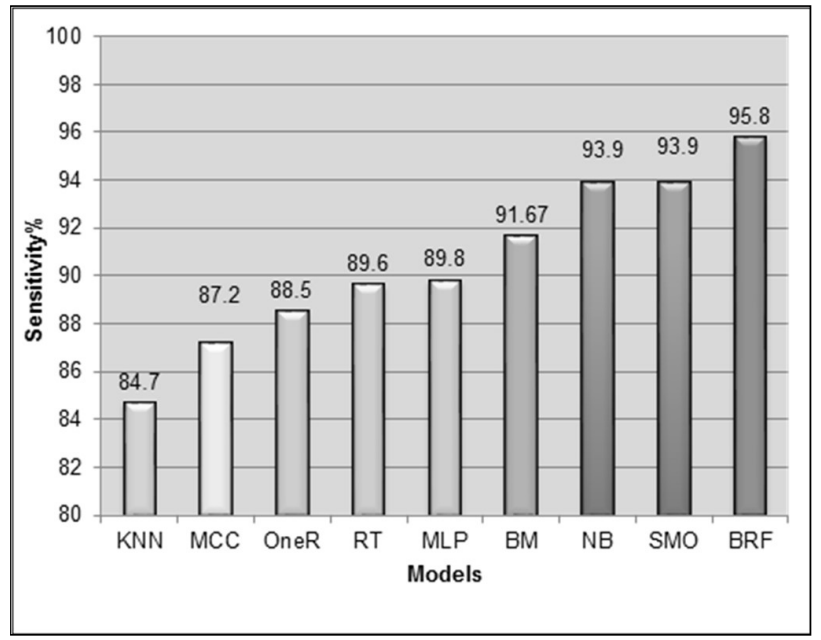

Fig. 3: Performance of BRF sensitivity compared to the eight ML models

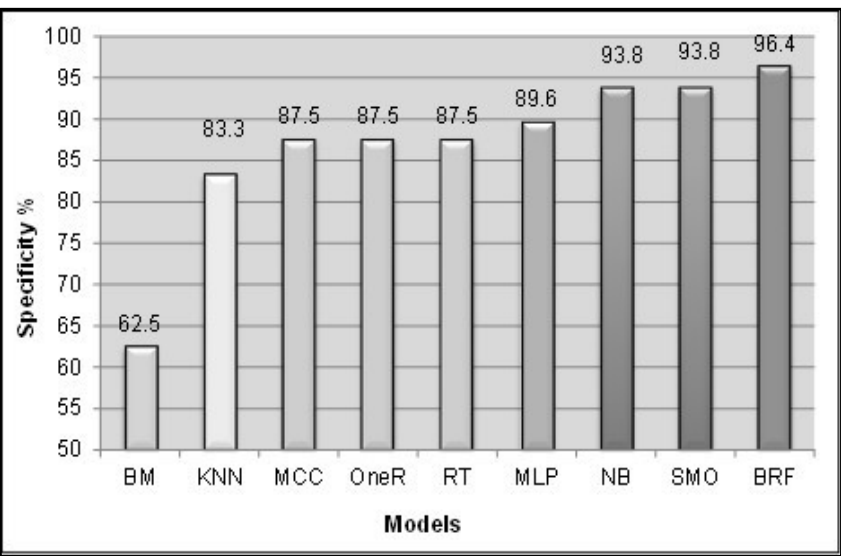

Fig. 4: Performance of BRF specificity compared to the eight ML models

From the experiments, the study found that BRF was able to obtain higher accuracy for OCDS. BRF was also observed to be the best model for classifying OCDS. Since BM was unable to classify most of Stage-Three (III) OCDS, an enhanced Bayesian method was proposed in this study (BRF) to improve the disease diagnosis model.

\subsection{CONCLUSION}

The purpose of this research is to recommend a novel diagnostic model that can assist users in making effective decisions regarding the primary stage of oral cancer using intelligent computing methods. In this study, a diagnosis inference engine was enhanced using the Bayesian Relevance Feedback Model (BRF) to generate the differential diagnosis for supporting the diagnosis of cancer stages. Its ability to eliminate non-diagnosis by removing zero posterior probabilities in the oral cancer stage proves that the BRF is a good model for oral cancer diagnosis. OCDS was divided into training and verification tests to obtain the validity and accuracy of the proposed approach for new cases. Eight different ML experiments were conducted to classify the cancer stage; Bayesian Model, K-Nearest Neighbor, Meta MultiClass Classifier, Rule OneR, Random Tree, Multilayer Perceptron, Naive Bayes, and SMOPoly Kernel. The experimental works were conducted using WEKA open source data mining software with 10-fold cross validation. The performance measure of the models was evaluated based on four different measures: accuracy, error rate, sensitivity, and specificity.

The finding of the experimental works demonstrates that adopting the proposed BRF for OCDS yielded a mean overall accuracy of $95.83 \%$ for the classifier algorithm. This shows that the BRF significantly achieved better precision results than the Bayesian model and the other eight ML algorithms. These results also clearly demonstrate 
that the proposed diagnostic model yielded increased classification accuracy in the diagnosis of clinical data. Thus, better diagnosis was achieved, which will assist less-experienced medical officers in giving an early oral cancer diagnosis. An obstacle in this research was that the experiments were not conducted with real users, such as patients with cancer, as it would require a much bigger effort to include real users compared to simply testing models using a dataset. Thus, additional numbers of oral cancer dataset feature and testing of the model with real users will be a part of our future work. Another direction for future studies is to consider BRF experimental work with various datasets and other new powerful classification models such as the deep Convolutional Neural Network (CNN).

\section{ACKNOWLEDGMENT}

We would like to thank all team members and contributors for the moral and technical support. Thank you also to the anonymous reviewers of this study. Their comments have played a significant role in improving the quality of this research.

\section{REFERENCES}

[1] R. C. Borra et al., "Development of an Open Case-Based Decision-Support System for Diagnosis in Oral Pathology", European Journal of Dental Education, Vol. 11, No. 2007, pp. 87-92.

[2] C. Hambleton et al., "Appropriate and Accurate Diagnosis of Thyroid Nodules: A Review of Thyroid FineNeedle Aspiration", International Journal of Clinical and Experimental Medicine (IJCEM), Vol. 6, No. 6, 2013, pp. 413-422.

[3] C. Y. Wang et al., "Predicting Survival of Individual Patients with Esophageal Cancer by Adaptive NeuroFuzzy Inference System Approach", Applied Soft Computing, Vol. 35, No. 2015, pp. 583-590.

[4] S. B. Edge et al., American Joint Committee on Cancer (AJCC) Cancer Staging Manual, 7, Springer, 2010.

[5] E. Al-Sukhni et al., "Diagnostic Accuracy of MRI for Assessment of T Category, Lymph Node Metastases, and Circumferential Resection Margin Involvement in Patients with Rectal Cancer: A Systematic Review and Meta-Analysis", Annals of Surgical Oncology, Vol. 19, No. 7, 2012, pp. 2212-2223.

[6] F. Amato et al., "Artificial Neural Networks in Medical Diagnosis", Journal of Applied Biomedicine, Vol. 11, No. 2, 2013, pp. 47-58.

[7] C. Castaneda et al., "Clinical Decision Support Systems for Improving Diagnostic Accuracy and Achieving Precision Medicine", Journal of clinical bioinformatics, Vol. 5, No. 1, 2015, pp. 4.

[8] A. Esteva et al., "Dermatologist-Level Classification of Skin Cancer with Deep Neural Networks", Nature, Vol. 542, No. 7639, 2017, pp. 115.

[9] F. Jia et al., "Deep Neural Networks: A Promising Tool for Fault Characteristic Mining and Intelligent Diagnosis of Rotating Machinery with Massive Data", Mechanical Systems and Signal Processing, Vol. 72, No. 2016, pp. 303-315.

[10] W. C. Wang et al., "Improving Forecasting Accuracy of Annual Runoff Time Series Using ARIMA Based On EEMD Decomposition", Water Resources Management, Vol. 29, No. 8, 2015, pp. 2655-2675.

[11] E. Turban et al., Decision Support Systems and Intelligent Systems, Prentice-Hall, 2007.

[12] J. J. Lee et al., "Bayesian Clinical Trials in Action", Statistics in Medicine, Vol. 31, No. 25, 2012, pp. $2955-$ 2972.

[13] S. K. Gupta, "Use Of Bayesian Statistics in Drug Development: Advantages and Challenges", International Journal of Applied and Basic Medical Research, Vol. 2, No. 1, 2012, pp. 3-6. 
[14] J. M. Guerrero et al., "Diagnosis of Cognitive Impairment Compatible with Early Diagnosis of Alzheimer's disease: A Bayesian Network Model based on the Analysis of Oral Definitions of Semantic Categories", Methods of Information in Medicine, Vol. 55, No. 1, 2016, pp. 42-49.

[15] A. Oniśko et al., "Impact Of Precision of Bayesian Network Parameters on Accuracy of Medical Diagnostic Systems", Artificial Intelligence in Medicine, Vol. 57, No. 3, 2013, pp. 197-206.

[16] F. L. Seixas, et al., "A Bayesian Network Decision Model for Supporting the Diagnosis of Dementia, Alzheimer'S Disease and Mild Cognitive Impairment", Computers in Biology and Medicine, Vol. 51, 2014, pp. 140-158.

[17] Y. Wei et al., "Bayesian Multivariate Meta-Analysis with Multiple Outcomes", Statistics in Medicine, Vol. 32, No. 17, 2013, pp. 2911-2934.

[18] C. D. Ferreira et al., "Relevance Feedback Based On Genetic Programming for Image Retrieval", Pattern Recognition Letters, Vol. 32, No. 1, 2011, pp. 27-37.

[19] E. D. Ves et al., "A Novel Bayesian Framework for Relevance Feedback in Image Content-Based Retrieval Systems", Pattern Recognition, Vol. 39, No. 9, 2006, pp. 1622-1632.

[20] L. Jiang et al., "Zero-Example Event Search Using Multimodal Pseudo Relevance Feedback", in Proceeding of the Proceedings of International Conference on Multimedia Retrieval, Glasgow, Scotland, 2014, pp. 297.

[21] J.H. Su et al., "Efficient Relevance Feedback for Content-Based Image Retrieval by Mining User Navigation Patterns", IEEE Transactions on Knowledge and Data Engineering, Vol. 23, No. 3, 2011, pp. 360-372.

[22] Y. Yang et al., "A Multimedia Retrieval Framework Based On Semi-Supervised Ranking and Relevance Feedback", IEEE Transactions on Pattern Analysis and Machine Intelligence, Vol. 34, No. 4, 2012, pp. $723-742$

[23] Z. A. Bakar et al., "Demographic Profile of Oral Cancer Patients in East Coast of Peninsular Malaysia", The International Medical Journal, Vol. 20, No. 3, 2013, pp. 362-364.

[24] F. Mohd et al., "Data Preparation for Pre-processing on Oral Cancer Data Set.", in Proceeding of the 13th International Conference on Control, Automation and Systems (ICCAS2013), Gwangju, Korea, 2013, pp. $324-328$.

[25] J. M. Guerrero et al., "Diagnosis of Cognitive Impairment Compatible with Early Diagnosis of Alzheimer's Disease: A Bayesian Network Model based on the Analysis of Oral Definitions of Semantic Categories", Methods of Information in Medicine, Vol. 55, No. 1, 2016, pp. 42-49.

[26] F. Calle-Alonso et al., "Computer-Aided Diagnosis System: A Bayesian Hybrid Classification Method", Computer Methods and Programs in Biomedicine, Vol. 112, No. 1, 2013, pp. 104-113.

[27] S. Suebnukarn et al., "A Bayesian Decision Support Model for Assessment of Endodontic Treatment Outcome", Oral Surgery, Oral Medicine, Oral Pathology, Oral Radiology, and Endodontology, Vol. 106, No. 3, 2008, pp. 48-58.

[28] M. D. Weinberg, "Computational Statistics Using the Bayesian Inference Engine", Monthly Notices of the Royal Astronomical Society, Vol. 434, No. 2, 2013, pp. 1736-1755.

[29] J. B. Tenenbaum et al., "Theory-based Bayesian Models of Inductive Learning and Reasoning", Trends in Cognitive Sciences, Vol. 10, No. 7, 2006, pp. 309-318.

[30] A. Stojadinovic et al., "Development of a Clinical Decision Model for Thyroid Nodules", BMC Surgery, Vol. 9, No. 1, 2009, pp. 12. 
[31] Nissan et al., "Predictive Model of Outcome of Targeted Nodal Assessment in Colorectal Cancer", Annals of Surgery, Vol. 251, No. 2, 2010, pp. 265-274.

[32] M. Thangaraj et al., "A Rule Based Decision Support System for Aiding Vitamin D Deficiency Management", Indian Journal of Science and Technology, Vol. 7, No. 1, 2014, pp. 48-542.

[33] N. Best et al., "A Bayesian Approach to Complex Clinical Diagnoses: A Case-Study in Child Abuse", Journal of the Royal Statistical Society. Series A: Statistics in Society, Vol. 176, No. 1, 2013, pp. 53-96.

[34] A. Pandey et al., "Stage Determination of Oral Cancer Using Neurofuzzy Inference System", in Proceeding of the 2014 IEEE Students' Conference on Electrical, Electronics and Computer Science (SCEECS), Bhopal, India, 2014, pp. 1-5.

[35] G. Kose et al., "Comparison of Different Inference Algorithms for Medical Decision Making", International Journal of Computational Intelligence Systems, Vol. 7, No. sup1, 2014, pp. 29-44.

[36] X. Yang et al., "Bayesian-Inference-Based Recommendation in Online Social Networks", Parallel and Distributed Systems, IEEE Transactions on, Vol. 24, No. 4, 2013, pp. 642-651.

[37] W. Neville et al., Oral and Maxillofacial Pathology, China, Saunders Elsevier, 2009.

[38] X. Zhou et al., "Development of Traditional Chinese Medicine Clinical Data Warehouse for Medical Knowledge Discovery and Decision Support", Artificial Intelligence in Medicine, Vol. 48, No. 2, 2010, pp. 139-152.

[39] W. Neville et al., "Oral Cancer and Precancerous Lesions", CA: A Cancer Journal for Clinicians, Vol. 52, No. 4, 2002, pp. 195-215.

[40] S. G. Patel et al., "TNM Staging of Cancers of the Head and Neck: Striving for Uniformity Among Diversity", CA: A Cancer Journal for Clinicians, Vol. 55, No. 4, 2005, pp. 242-258.

[41] A. Omar, "The Outline of Prognosis and New Advances in Diagnosis of Oral Squamous Cell Carcinoma (OSCC): Review of the Literature", Journal of Oral Oncology, Vol. 2013, No. 2013, 2013, pp. 1-13.

[42] R. Trotta, "Applications of Bayesian Model Selection to Cosmological Parameters", Monthly Notices of the Royal Astronomical Society, Vol. 378, No. 1, 2007, pp. 72-82.

[43] R. H. Walker, "Differential Diagnosis of Chorea", Current Neurology and Neuroscience Reports, Vol. 11, No. 4, 2011, pp. 385-395.

[44] W. Zhu et al., "Sensitivity, Specificity, Accuracy, Associated Confidence Interval and ROC Analysis with Practical SAS Implementations", in Proceeding of the NESUG, Baltimore, Maryland, 2010, pp. 1-9.

[45] K. J. Drobatz, "Measures of Accuracy and Performance of Diagnostic Tests", Journal of Veterinary Cardiology, Vol. 11, Supplement 1, No. 2009, pp. S33-S40.

[46] P. Eusebi, "Diagnostic Accuracy Measures", Cerebrovascular Diseases, Vol. 36, No. 4, 2013, pp. $267-272$. 